
\title{
R Research Square \\ Effect of ambient air pollutants and meteorological factors on COVID-19 transmission
}

\section{Ying Jiang}

Shanghai Chang Zheng Hospital

Xiao-Long Xu

Shanghai Chang Hai Hospital

Da-Wei Dai

Shanghai Chang Zheng Hospital

Xiao-Jun Wu

Shanghai Cancer Center

Jun-Yu Wang

Shanghai Chang Zheng Hospital

Yun-Kun Wang

Shanghai Chang Zheng Hospital

Zhen-Yu Huang

Shanghai Chang Zheng Hospital

Guang-Ming Wang

Shanghai Chang Zheng Hospital

Yan-Jun Guan ( $\sim$ guanyjsigma@yeah.net )

https://orcid.org/0000-0001-6828-655X

Research article

Keywords: COVID-19, viral transmission, air pollution, meteorological factor, Wuhan

Posted Date: April 23rd, 2020

DOl: https://doi.org/10.21203/rs.3.rs-22227/v2

License: (c) (i) This work is licensed under a Creative Commons Attribution 4.0 International License.

Read Full License 
The authors have withdrawn this preprint from Research Square 